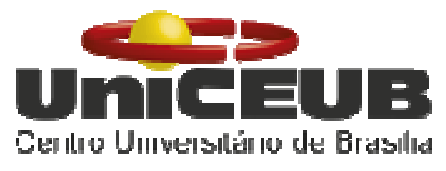

CENTRO UNIVERSITÁRIO DE BRASÍLIA - UnICEUB PROGRAMA DE INICIAÇÃO CIENTÍFICA

ANA CAROLINA SALAZAR ALBUQUERQUE

\title{
PROGRAMA DE AVALIAÇÃO E ACOMPANHAMENTO DO TRAUMA DE CABEÇA NO FUTEBOL
}

BRASÍLIA 


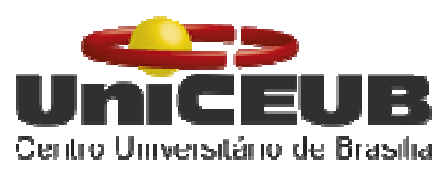

ANA CAROLINA SALAZAR ALBUQUERQUE

PROGRAMA DE AVALIAÇÃO E ACOMPANHAMENTO DO TRAUMA DE CABEÇA NO FUTEBOL

Relatório final de pesquisa de Iniciação Científica apresentado à Assessoria de Pós-Graduação e Pesquisa.

Orientação: Márcio de Paula Oliveira

BRASÍLIA 


\section{RESUMO}

A concussão cerebral é uma lesão muito comum na prática de esportes de contato e tem uma alta prevalência no futebol. Palavra derivada da palavra latina "concutere", que significa tremer violentamente, é um subtipo de traumatismo craniano frequentemente usado na literatura como sinônimo de traumatismo craniano leve. Com base no IV Consenso sobre Concussão Cerebral no Esporte realizado pelo Comitê Olímpico Internacional (COI) e pela Federação Internacional de Futebol (FIFA), a concussão cerebral pode resultar em alterações neuropatológicas e distúrbios funcionais com sintomas clínicos agudos, sem demonstrar uma lesão estrutural. Portanto, nenhuma alteração será identificada nos estudos tradicionais de neuroimagem. Dessa forma, a concussão é uma condição mal diagnosticada no Brasil e na América do Sul. Seu diagnóstico tem como base a investigação de sinais e sintomas clínicos. Embora muitas técnicas inovadoras demonstrem ser boas ferramentas complementares, nenhum marcador foi caracterizado como ótimo e deve ser adotado como padrão-ouro para detectar e acompanhar a evolução da concussão. Esse estudo objetiva estabelecer um programa sistematizado para concussão cerebral no futebol, pela primeira vez no Brasil.

Palavras-Chave: Concussão cerebral. Traumatismo craniano leve. Futebol. 


\section{Sumário}

Sumário 4

1. INTRODUÇÃO

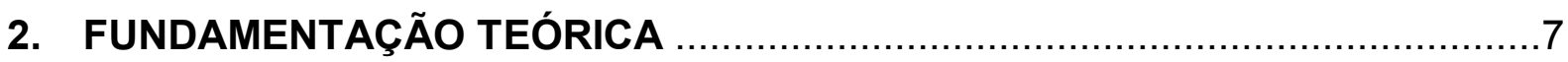

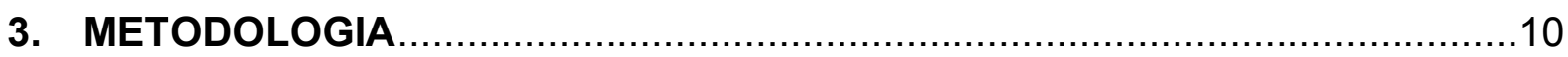

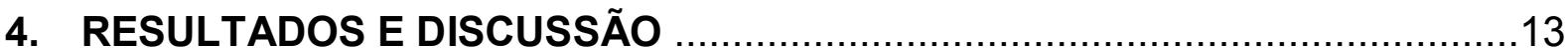

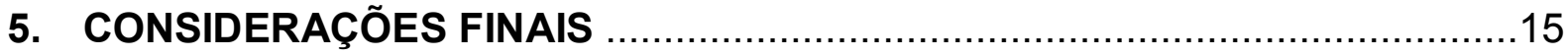

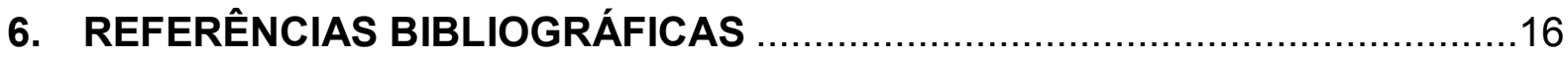

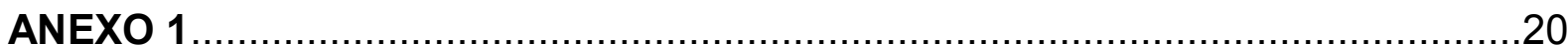

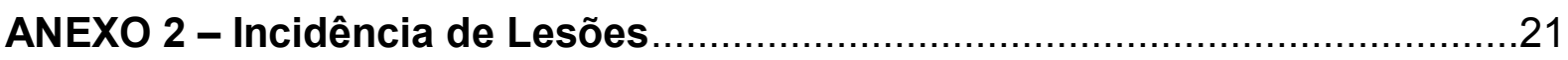




\section{INTRODUÇÃO}

A concussão cerebral é uma lesão muito comum na prática de esportes de contato e tem uma alta prevalência no futebol e outros esportes olímpicos. É um subtipo de traumatismo craniano frequentemente usado na literatura como sinônimo de traumatismo craniano leve. Pode causar alterações neuropatológicas, com sintomas clínicos agudos que refletem um distúrbio funcional e não uma lesão estrutural. Portanto, nenhuma alteração será identificada nos estudos tradicionais de neuroimagem" (MCCRORY et al 2013).

Atualmente, o diagnóstico de concussão cerebral baseia-se na investigação de sinais e sintomas clínicos, devendo abranger diferentes domínios incluindo sinais clínicos, sintomas, comprometimento cognitivo, alterações de humor e comportamento. Quando um ou mais sintomas são observados, deve-se suspeitar de concussão. Neste caso, o atleta deve ser retirado da prática esportiva no dia da lesão e um programa de tratamento e acompanhamento de ser estabelecido imediatamente (MCCRORY et AL, 2013).

Pensando nisso foram desenvolvidas ferramentas para esse diagnóstico inicial. A Ferramenta de Reconhecimento de Concussão (CRT) e ferramenta de avaliação de concussão no esporte - versão 5 (SCAT 5) auxiliam no diagnóstico de concussão cerebral aguda (MCCRORY et al 2013). Os testes cognitivos computadorizados também devem ser utilizados para auxiliar no diagnóstico mesmo em atletas assintomáticos.

Com a concussão clinicamente confirmada, o atleta não deve retornar ao esporte no mesmo dia, devendo retornar as atividades esportivas apenas quando a condição já tiver sido tratada e os processos fisiopatológicos resolvidos. Com isso, um programa de retorno ao esporte deve ser seguido e respeitado. Ser assintomático em repouso, em atividades físicas e cognitivas extenuantes são condições mínimas necessárias para caracterizar uma resolução da concussão (MCCRORY et al 2013). Porém, existem algumas dificuldades em relação ao tratamento e tomada de decisão no retorno do atleta ao esporte. Em muitos casos os atletas assintomáticos apresentam uma diminuição da função cerebral que não é clinicamente detectável (SANDEL et al 2013). Apresentando constatação diagnóstica complexa, a concussão é uma condição mal diagnosticada no Brasil e na América do Sul (MCCRORY et al, 2008).

A falta de critérios objetivos clinicamente detectáveis também coloca o médico em risco de ser influenciado por atletas que, mesmo sintomáticos, omitem seus sintomas com o objetivo de retornar ao esporte o mais cedo possível.

Para evitar consequências fatais, como síndrome de segundo impacto ou encefalopatia traumática crônica devido a concussão repetidas, recomenda-se que os profissionais de saúde acompanhem os atletas de modalidades de contato que tenham treinamento de identificação e tratamento de concussão.

O objetivo desse estudo foi estabelecer um programa sistematizado para concussão cerebral, abordando a educação continuada dos profissionais e atletas e o acompanhamento de jogadores profissionais de futebol, desde o diagnostico até o retorno ao esporte, de forma replicável para outros clubes de futebol no Brasil e em outros países em desenvolvimento, obtendo escores basais e pós-traumáticos de testes cognitivos computadorizados.

Ao mesmo tempo em que a atividade física realizada pelos atletas é benéfica, pode trazer consequências severas aos praticantes, sejam elas físicas, sociais, econômicas ou psicológicas. Os jogadores estão frequentemente sujeitos a riscos dentro de sua prática esportiva. Além disso, os jogadores de futebol profissional 
dependem na maioria das vezes do esporte para sua sobrevivência. Logo, o afastamento traz aos atletas consequências sócio-econômicas negativas. Com um bom preparo voltado a avaliação, reabilitação e prevenção, estes riscos podem ser minimizados, favorecendo um retorno ao esporte mais seguro. Assim, esse trabalho se torna relevante por poder fornecer maiores esclarecimentos acerca deste tema. Se trata de um projeto nunca realizado previamente no Brasil. 


\section{FUNDAMENTAÇÃO TEÓRICA}

Atualmente no Brasil são 730 times de futebol profissional, abrangendo 19.000 atletas profissionais. Nesse contexto, são esperadas 800 concussões por ano em jogos profissionais. Apesar da incidência alarmante, a maioria dos times não aplica seus recursos em cuidados à saúde. Nenhum desenvolveu ainda um programa sistemático para gerenciar situações que envolvem concussão (NETO et al, 2019).

Um estudo epidemiológico realizado nos Jogos Olímpicos de 2004 mostrou que a cabeça era o segundo local com maior incidência de lesões, correspondendo a $24 \%$ de todos os incidentes relatados. Cerca de $10 \%$ de todos as lesões na cabeça foram concussão cerebral (JUNGE et al 2006).

A associação universitária de atletas identificou que entre as 3.825 lesões sofridas pela prática de futebol nos anos de 2009 a 2015, cerca de $3 \%$ eram Concussões Cerebrais (ROSS et al 2016).

Cerca de $36 \%$ dos jogadores de pólo aquático de alto desempenho já sofreram em média 2 episódios de Concussão Cerebral (ROBERT et al 2016).

Uma recente revisão sistemática identificou 199 estudos científicos sobre epidemiologia de lesões no rugby. A revisão indicou que Concussão Cerebral corresponde a $29 \%$ de todas as lesões em movimentos ilegais e $9 \%$ sofridas em lances legais ( $\mathrm{KOH}$ et al 2014).

A concussão, uma palavra derivada da palavra latina "concutere", que significa tremer violentamente, é um subtipo de traumatismo craniano frequentemente usado na literatura como sinônimo de traumatismo craniano leve. Com base no IV Consenso sobre Concussão Cerebral no Esporte realizado pelo Comitê Olímpico Internacional (COI) e pela Federação Internacional de Futebol (FIFA), a concussão cerebral pode resultar em alterações neuropatológicas, com sintomas clínicos agudos que refletem um distúrbio funcional e não uma lesão estrutural. Portanto, nenhuma alteração será identificada nos estudos tradicionais de neuroimagem (MCCRORY et al 2013).

Atualmente, o diagnóstico de concussão cerebral baseia-se na investigação de sinais e sintomas clínicos. Embora muitas técnicas inovadoras demonstrem ser boas ferramentas complementares, nenhum marcador foi caracterizado como ótimo e deve ser adotado como padrão-ouro para detectar e acompanhar a evolução da concussão. Apresentando constatação diagnóstica complexa, a concussão é uma condição mal diagnosticada no Brasil e na América do Sul (MCCRORY et al, 2008).

A abordagem médica da concussão deve abranger diferentes domínios, incluindo sintomas (cefaleia, alterações visuais e náuseas), sinais clínicos (perda da consciência, fadiga, distúrbios do equilíbrio, fala e coordenação motora), comprometimento cognitivo (amnésia, dificuldade de concentração e reação iniciar ou manter o sono ou sentir muito sono e dormir mais que o habitual), alterações de humor e comportamento (depressão, irritabilidade, agressividade e apatia). Quando um ou mais sintomas são observados, deve-se suspeitar de concussão. Neste caso, o atleta deve ser retirado da prática esportiva no dia da lesão e um programa de tratamento e acompanhamento de ser estabelecido imediatamente (MCCRORY et $\mathrm{AL}, 2013)$.

Pensando nisso, foram desenvolvidas ferramentas para esse diagnóstico inicial, a Ferramenta de Reconhecimento de Concussão (CRT) e a ferramenta de avaliação de concussão no esporte- versão 5 (SCAT 5) com sua versão para crianças (Criança SCAT5) ajudam no diagnóstico de concussão cerebral aguda abordando os principais sinais, sintomas e funções cognitivas associadas a lesão (MCCRORY et al 2013). 
A investigação dos sintomas, assim como os testes cognitivos breves utilizados no SCAT 5, apresentam boa sensibilidade e especificidade para o diagnóstico de concussão e recomenda-se que seja feita uma avaliação com as mesmas ferramentas antes da concussão, para que uma comparação possa ser feita entre os escores pré e pós (GIZA et al 2013; MCCRORY et al 2013).

Apesar dos sintomas clínicos apresentarem alta sensibilidade nas detecções de concussão, os testes cognitivos computadorizados podem ser utilizados para auxiliar no diagnóstico mesmo em atletas assintomáticos.

Segundo a Academia Americana de Neurologia, a tomografia computadorizada (TC) não devem ser usada para diagnosticar concussão, mas sim para investigar e detectar lesões mais graves na cabeça (hemorragias, amnésias traumáticas, distúrbios neurológicos focais ou sinais de deterioração clínica) (GIZA et al 2013).

A ressonância magnética funcional apresenta resultados que se correlacionam com os sintomas e a recuperação clínica do paciente e pode ser usada como recurso de monitoramento adicional quando disponível (CHEN et al 2007).

Com a concussão clinicamente confirmada, o atleta não deve retornar ao esporte no mesmo dia, devendo retornar as atividades esportivas apenas quando a condição já tiver sido tratada e os processos fisiopatológicos resolvidos. Com isso, um programa de retorno ao esporte deve ser seguido e respeitado. Ser assintomático em repouso, em atividades físicas e cognitivas extenuantes são condições mínimas necessárias para caracterizar uma resolução da concussão (MCCRORY et al 2013). Existem algumas dificuldades em relação ao tratamento e tomada de decisão no retorno do atleta ao esporte. Em muitos casos os atletas assintomáticos apresentam uma diminuição da função cerebral que não é clinicamente detectável (SANDEL et al 2013).

A falta de critério objetivos clinicamente detectáveis também coloca o médico em risco de ser influenciado por atletas que, mesmo sintomáticos, omitem seus sintomas com o objetivo de retornar ao esporte o mais cedo possível.

Para evitar consequências fatais, como síndrome de segundo impacto ou encefalopatia traumática crônica devido a concussão repetidas, recomenda-se que os profissionais de saúde acompanhem os atletas de modalidades de contato que tenham treinamento de identificação e tratamento de concussão.

Para acompanhar a recuperação e confirmar que o processo químico desencadeado pelo traumatismo craniano "foi resolvido", algumas ferramentas estão se mostrando eficazes, principalmente quando aplicadas em uma avaliação pré e pós concussão. Os escores dessas avaliações devem ter retornado aos valores individuais antes de retornar a prática esportiva.

O SCAT 5 é uma ferramenta gratuita que auxilia no acompanhamento do atleta com concussão e recomenda-se que seja preenchido também pré-temporada (MCCRORY et al 2013).

Testes cognitivos computadorizados têm sido estudados há muitos anos e possuem extensa validação cientifica para esse fim (MCCRORY et al 2013). Testes computadorizados e também tradicionais (papel e caneta) têm sido utilizados por muitas instituições esportivas amadoras e profissionais como uma ferramenta obrigatória para o acompanhamento, até que os resultados sejam normalizados.

Muitas estratégias foram avaliadas para prevenir a concussão cerebral (EMERY et al 2017). Estudos têm demonstrado que programas de fortalecimento de pescoço podem reduzir o risco de sustentar lesões cervicais e concussões na pratica de futebol americano e rúgbi (JIN et al 2017, HRYSONMALLIS et al 2016). 
Ainda faltam dados prospectivos para adotar tais praticas com o objetivo de prevenir concussão em jogadores de futebol.

A educação sobre concussão tem sido a prioridade maior para a Confederação Brasileira de Futebol nos últimos anos, porém ainda não foi transformado em programas sistemáticos pelos médicos dos times. Atletas profissionais e amadores continuam desinformados e subdiagnosticados (NETO et al, 2019). 


\section{METODOLOGIA}

\subsection{Tipo de estudo e delineamento}

Foi realizado um estudo prospectivo, longitudinal e controlado com atletas de 18 a 35 anos federados a Federação Brasiliense de Futebol (FBF).

\subsection{Amostra}

Para compor a amostra, convidamos os 12 clubes da primeira divisão do Campeonato Candango. Foram excluídos do estudo aqueles que perderam alguma das avaliações, abandonaram a pesquisa no período de intervenção ou que apresentaram algum sintoma exacerbado antes do início do programa. Dessa forma, cinco times foram excluídos, não se propondo a realizar as etapas necessárias.

Os 7 (sete) clubes restantes cederam, por conveniência, seus atletas a realizarem os procedimentos da pesquisa. Foram avaliados 187 atletas, idade média de 25.2 (5.1), do gênero masculino, federados à FBF e que assinaram o Termo de Consentimento Livre e Esclarecido para participar do estudo.

\subsection{Coleta de dados}

\subsubsection{Local da coleta}

Todas as avaliações foram realizadas no Hospital HOME - Centro Médico de Excelência FIFA.

\subsubsection{Procedimentos}

3.3.2.1. Educação do Departamento Médico dos times de

Futebol

Inicialmente, foi realizado um programa de educação presencial para profissionais nos Departamentos Médicos de todos os clubes participantes. A propedêutica diagnóstica foi abordada de maneira teórica e prática, bem como os métodos de tratamento e controle da concussão cerebral no esporte. Além disso, a metodologia do estudo foi apresentada de forma clara com o intuito de esclarecer questões sobre a pesquisa e estabelecer uma comunicação contínua entre os pesquisadores e a comissão técnicas dos clubes.

\subsubsection{Avaliação pré-temporada}

Um portal da web foi utilizado para inserir todas as informações sobre a pesquisa, registrando os dados das avaliações basais de cada atleta na plataforma online Ubrain. Foi realizado o cadastro de cada participante, determinando um número de identificação e login seguro para responder aos questionários e testes cognitivos.

Após realizado o cadastro, os atletas responderam a um questionário contendo uma escala de sintomas (englobando dor de cabeça, pressão na cabeça, 
dor no pescoço, náusea ou vômito, tontura, visão borrada, problemas de equilíbrio, sensibilidade à luz, sensibilidade ao ruído, sensação de lentificação, sensação de "nublado", dificuldade em se concentrar, dificuldade com a memória, fadiga ou pouca energia, confusão, sonolência, dificuldade para pegar no sono, mais emotivo, irritabilidade, triste, nervoso ou ansioso), prova índex nariz e o teste de equilíbrio BESS. Na prova índex nariz, o participante estende o membro superior e logo em seguida o flexiona, toca com o dedo indicador a ponta do nariz. No BESS (Balance Error Scorring System) modificado, os atletas foram testados em equilíbrio unipodal (em pé na perna não dominante e mãos no quadril), devendo manter essa postura por 20 segundos de olhos fechados em uma superfície estável. Foi registrado o número de "erros". Esse teste foi utilizado para a avaliação do equilíbrio por identificar déficits de estabilidade postural. Um erro é definido como abrir os olhos, levantar as mãos do quadril, pisar, tropeçar ou sair da posição, levantar o antepé ou o calcanhar ou abduzir o quadril em mais de 30 graus.

Em seguida cada atleta realizou quatro testes cognitivos informatizados. Cada teste foi exibido no computador na janela do navegador da web e os resultados foram salvos automaticamente no mesmo portal da web onde os outros dados foram inseridos. Os participantes foram solicitados a minimizar as distrações durante os testes. Para cada tarefa, uma apresentação e informações de resumo foram exibidas no meio da tela, seguidas por uma fase de familiarização e depois pela tarefa. Um som de feedback foi utilizado para diferenciar respostas corretas e incorretas. Respostas feitas antes que o estímulo apareça ou mais rápido que $95 \mathrm{~ms}$ foram definidas como antecipatórias. Estimativas em adultos para confiabilidade do teste de re-teste para tarefas com os mesmos princípios estão entre 0,84 e 0,91 (FALLETI et al 2006; CAIRNEY et al 2003). Cada tarefa foi descrita em sua ordem de apresentação, conforme descrito abaixo:

- Tarefa simples de tempo de reação (TRS): é uma tarefa simples de tempo de reação que mede a função psicomotora. Nesta tarefa, os sujeitos foram solicitados a pressionar a tecla "J" o mais rápido possível quando o alvo surgisse na tela. Foram executadas 35 respostas em cada teste. $O$ tempo gasto entre a apresentação do estímulo e a resposta do atleta foi medido pelo sistema;

- Teste de reação de escolha (CRT): é uma tarefa de escolha de tempo de reação que requer atenção visual. Essa tarefa é semelhante à tarefa anterior, porém os participantes foram instruídos a pressionar a tecla "J" se o destino exibido fosse vermelho e " $\mathrm{F}$ " se o destino exibido fosse azul. $O$ teste termina depois que 30 alvos são exibidos. A precisão das respostas, bem como o tempo médio gasto digitando a chave correta após o estímulo foram computados;

- Teste de memória episódica (EMT): é uma tarefa contínua de aprendizagem e reconhecimento que testa o reconhecimento visual episódico de memória e atenção. Nesta tarefa, os participantes foram instruídos a responder com a tecla "J" se o cartão que apareceu anteriormente aparecesse e "F" se o cartão não apareceu anteriormente. Letras com diferentes cores, números e símbolos foram exibidas. Há letras que foram repetidas várias vezes de forma aleatória e letras repetidas apenas uma vez em 88 testes; 
- Teste de Memória de Trabalho (WMT): avalia a memória de trabalho e a atenção. É um tradicional back test. Os participantes foram instruídos a responder "J" se o cartão exibido fosse exatamente o cartão anterior ou "F" se o cartão exibido não correspondesse ao cartão anterior. Os cartões usados foram diferentes daqueles da EMT para evitar confusão e foram necessárias 31 respostas para completar a tarefa.

Foi realizada também uma avaliação do desempenho muscular cervical utilizando dinamômetro manual, segundo metodologia descrita por Theo Versteegh (VERSTEEGH et al 2013).

Os procedimentos descritos acima foram realizados na pré temporada, para estabelecer escores basais.

\subsubsection{Acompanhamento}

Durante toda a temporada, as lesões ocorridas foram documentadas pela equipe de pesquisa, bem como os casos de concussão cerebral.

\subsubsection{Avaliação pós concussão}

Os atletas que tiveram o diagnóstico de concussão realizaram o SCAT 5, o CRT (utilizado para assistência diagnóstica no momento do trauma e afastamento do atleta da partida), tomografia computadorizada de crânio (para descartar lesões cerebrais mais graves) e passaram por uma avaliação com médico especialista. Além disso repetiram os questionários e testes cognitivos informatizados. 


\section{RESULTADOS E DISCUSSÃO}

Participaram da pesquisa 7 dos 12 times profissionais locais, totalizando 187 atletas com idade média de 25.2 (5.1). Na avaliação inicial $24,6 \%$ dos atletas relataram que já haviam tido pelo menos uma concussão prévia, corroborando com o estudo de JUNGE et al, 2006, ROBERT et al, 2016 e KOH et AL, 2014, que demonstram alta prevalência de concussão cerebral em esportes de contato. Além disso, de todos os atletas que tiveram concussão prévia, somente um teve o diagnóstico fechado, corroborando com o trabalho de ROWE et AL, 2017, no qual se conclui que muitos casos de concussão são mal diagnosticados, trazendo grandes prejuízos pela falta de acompanhamento e tratamento adequados. Segue em anexo a tabela com a relação da porcentagem de concussão por atleta (ANEXO 1).

Foram documentadas 75 lesões ocorridas no Campeonato Candango no período de janeiro a abril de 2019, em um total de 46 jogos. Segue em anexo tabela sobre a incidência das lesões (ANEXO 2).

Em relação à concussão, ocorreram quatro casos de concussão, porém apenas dois foram diagnosticados. Dois casos de trauma de cabeça referiram sinais e sintomas característicos de concussão, porém seus médicos não acharam necessário fazer uma reavaliação e o cronograma de treinamento não sofreu alterações. Os dois atletas continuaram jogando na mesma partida. O jogador 1 reportou amnésia retrógrada, se sentiu lento, fadigado, com dor de cabeça leve e náusea (os sintomas perduraram por um dia). $\mathrm{O}$ jogador 2 relatou tortura e dores de cabeça que também se mantiveram por um dia.

Sobre os casos de concussão que tiveram diagnótico fechado: o jogador 1 é goleito e sofreu lesão dia 9 de fevereiro, no final do jogo. Teve perda de consciência por alguns segundos, sentiu fadiga, confusão e problemas de concentração. Foi realizada um tomografia computadorizada e o resultado foi normal. No $2^{\circ}$ dia pós lesão não relatou sintomas, na bateria de testes cognitivos obteve resultados normais e seguiu o protocolo para voltar a jogar. Segue abaixo o gráfico da análise de mudança ao longo do tempo, desde a primeira avaliação até dois dias após a ocorrência da lesão.

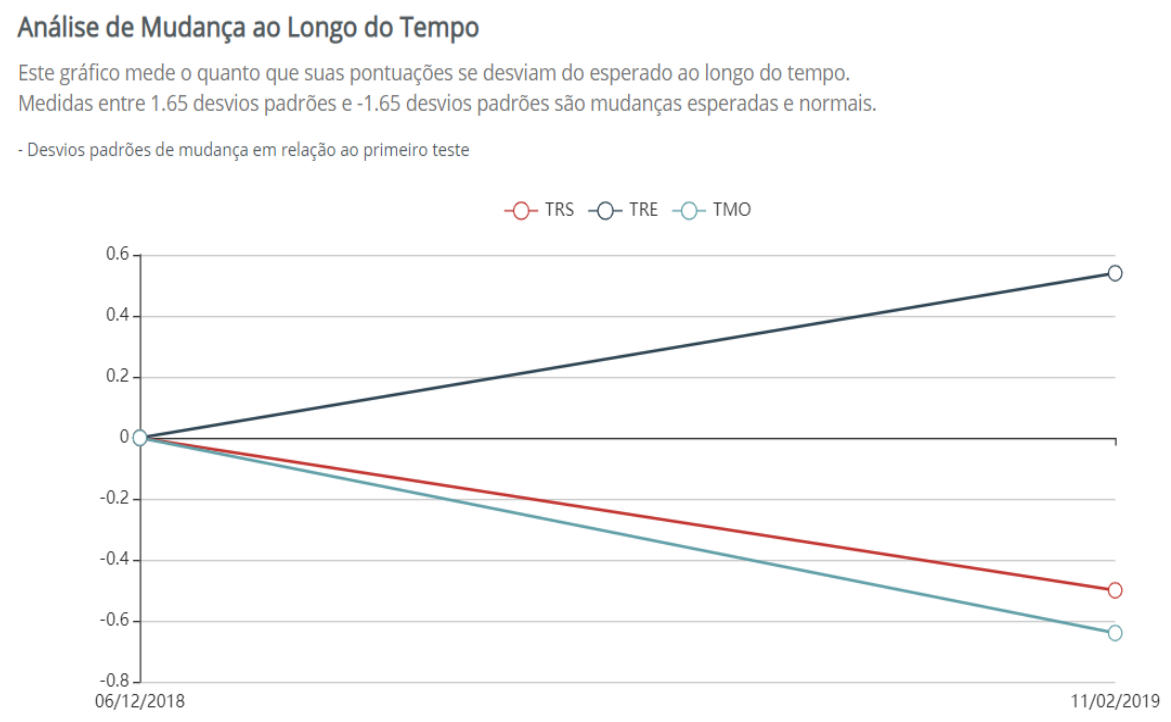

O jogador 2 também é goleiro, e teve perda de consciência por alguns segundos, fadiga, dor de cabeça, problemas de equilíbrio e problemas para dormir. 
Sua tomografia computadorizada se apresentou normal. No $2^{\circ}$ dia pós lesão não apresentou nenhum sintoma. A bateria de testes cognitivos se manteve anormal. No $3^{\circ}$ dia pós lesão continuou sem sintomas e nos testes cognitivos obteve resultados normais. Seguiu o protocolo de retorno ao jogo. Segue abaixo o gráfico da análise de mudança ao longo do tempo, desde a primeira avaliação até dois três após a ocorrência da lesão.

Análise de Mudança ao Longo do Tempo

Este gráfico mede o quanto que suas pontuações se desviam do esperado ao longo do tempo.

Medidas entre 1.65 desvios padrões e -1.65 desvios padrões são mudanças esperadas e normais.

- Desvios padrões de mudança em relação ao primeiro teste

$-O-$ TRS $-O-$ TRE $-O-$ TMO

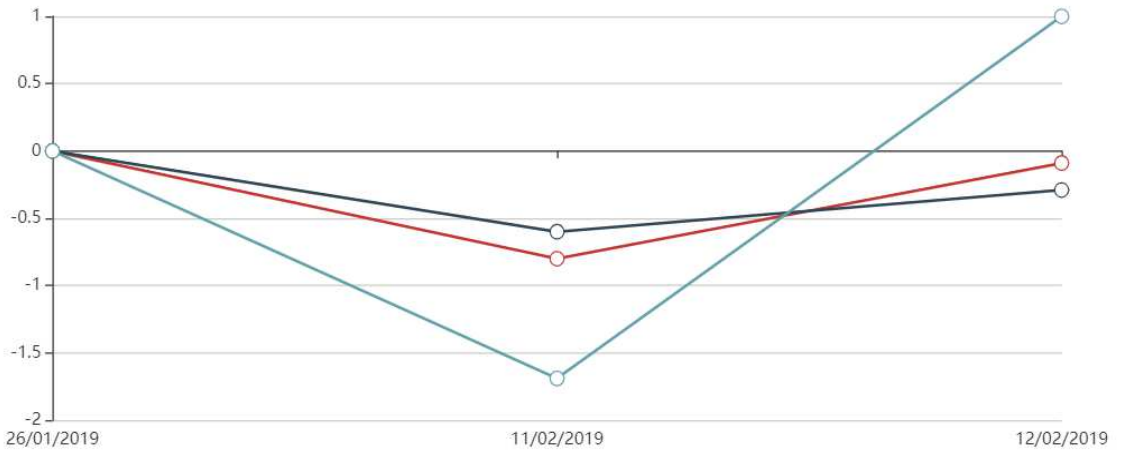




\section{CONSIDERAÇÕES FINAIS}

Além do trabalho ter atingido seus objetivos, abriu oportunidade para novas pesquisas em âmbitos menores. Estamos redigindo mais 4 artigos utilizando os dados desse estudo, sobre: a avaliação restrospectiva por interrogação dos 190 atletas sobre histórico de concussão; a correlação de avaliações cognitivas pré participativas dentro do programa de concussão cerebral com incidência de lesões ao longo de uma temporada de futebol; os dados normativos dos testes de força nos atletas de futebol profissionais; e a validação do método de avaliação de força cervical com o dinamômetro manual. Todos atualmente em andamento, sendo que para este último foi necessário realizar uma nova coleta de dados, já concluída e a qual atualmente já está em análise dos resultados.

Traçamos como meta para um futuro próximo, além das publicações referidas acima, aumentar o alcance desse projeto para outras regiões (inicialmente na região Norte); implementar programas de acompanhamento da concussão em mais times, especialmente nos que possuem menos recursos; e executar uma campanha nacional no Brasil para alertar a mídia, os atletas, familiares e diretores dos times sobre a importância de dar atenção à concussão. 


\section{REFERÊNCIAS BIBLIOGRÁFICAS}

Inklaar H. Soccer injuries. I: Incidence and severity. Sports Med 18: 55-73, 1994

Tucker AM. Common soccer injuries. Diagnosis, treatment and rehabilitation. Sports Med 23: 21-32, 1997

Matser JT, Kessels AG, Jordan BD, et al: Chronic traumatic brain injury in professional soccer players. Neurology 51: 791-796, 1998

Delaney JS, Lacroix VJ, Leclerc $S$, et al. Concussions among university football and soccer players. Clin J Sport Med. 2002;12:331-338

McCrory et al. Consensus Statement On Concussion In Sport - The 5th International Conference On Concussion In Sport Held In Berlin, October 2016. British Journal of Sports Medicine 2017 (available at www.bjsm.bmj.com)

Tamara, C et al. Representative Baseline Values on the Sport Concussion Assessment Tool 2 (SCAT2) in Adolescent Athletes Vary by Gender, Grade, and Concussion History.The American Journal of Sports Medicine. 2013.

Carey, DL et al. Modelling Training loads and injuries: the dangers of discretization. 2018.

Barros Junior, EA et al. Evaluation of the muscular strength of the flexors and extensors of the knees using the handheld dynamometer Lafayette ${ }^{\circledR}$ after anterior cruciate ligament reconstruction. Jan/ 2017.

David, R et al. Systematic Review of the Balance Error Scoring System. Feb/ 2015.

Makdissi, $M$ et al. Concussion in sport: best from Berlin, direction from Dublin and gems from gridiron. 2018.

Giza CC, Kutcher JS, Ashwal S, Barth J, Getchius TS, Gioia GA, Gronseth GS, Guskiewicz K, Mandel S, Manley G, McKeag DB, Thurman DJ, Zafonte R. Summary of evidence-based guideline update: evaluation and management of concussion in sports: report of the Guideline Development Subcommittee of the American Academy of Neurology. Neurology. 2013 Jun 11;80(24):2250-7;

Buzas D, Jacobson NA, Morawa LG Concussions From 9 Youth Organized Sports: Results From NEISS Hospitals Over an 11-Year Time Frame, 2002-2012. Orthop J Sports Med. 2014 Apr 2;2(4);

Zhang AL, Sing DC, Rugg CM, Feeley BT, Senter C. The Rise of Concussions in the Adolescent Population. Orthop J Sports Med. 2016 Aug 16;4(8):2325967116662458. doi: 10.1177/2325967116662458. eCollection 2016 Aug;

McCrory $\mathrm{P}$, Meeuwisse $\mathrm{WH}$, Aubry $\mathrm{M}$, et al. Consensus statement on concussion in sport: the 4th International Conference on Concussion in Sport held in Zurich, November 2012. Br J Sports Med. 2013;47(5):250-258;

Junge A, Langevoort G, Pipe A, Peytavin A, Wong F, Mountjoy M, Beltrami G, Terrell R, Holzgraefe M, Charles R, Dvorak J. Injuries in team sport tournaments during the 2004 Olympic Games. Am J Sports Med. 2006 Apr;34(4):565-76;

Roos KG, Wasserman EB, Dalton SL, Gray A, Djoko A, Dompier TP, Kerr ZY.Br Epidemiology of 3825 injuries sustained in six seasons of National Collegiate Athletic Association men's and women's soccer (2009/2010-2014/2015). J Sports Med. 2016 May 17. pii: bjsports-2015-095718. doi: 10.1136/bjsports-2015-095718. [Epub ahead of print]; 
Robert S. Blumenfeld, Jessica C. Winsell, James W. Hicks, Steven L. Small. The Epidemiology of Sports-Related Head Injury and Concussion in Water Polo Front Neurol. 2016; 7: 98;

Gardner A, Iverson GL, Levi CR, Schofield PW, Kay-Lambkin F ${ }^{3}$, Kohler RM, Stanwell $\mathrm{P}$. A systematic review of concussion in rugby league. $\mathrm{Br} \mathrm{J}$ Sports Med. 2015 Apr;49(8):495-8;

Koh JO, Cassidy JD. Incidence study of head blows and concussions in competition taekwondo. Clin J Sport Med. 2004 Mar;14(2):72-9;

Chen J, Johnston K, Collie A, et al. A validation of the Post Concussion Symptom Scale in the assessment of complex concussion using cognitive testing and functionalMRI. J NeurolNeurosurg Psych. 2007;78:1231-1238;

Sandel NK, Lovell MR, Kegel NE, Collins MW, Kontos AP The relationship of symptoms and neurocognitive performance to perceived recovery from sports-related concussion among adolescent athletes. AppINeuropsychol Child. 2013;2(1):64-9;

Steven P. Broglio, Robert C. Cantu, Gerard A. Gioia, Kevin M. Guskiewicz, Jeffrey Kutcher, Michael Palm, Tamara C. Valovich McLeod National Athletic Trainers' Association Position Statement: Management of Sport Concussion J Athl Train. 2014 Mar-Apr; 49(2): 245-265;

Olivera A, Lejbman N, Jeromin A, French LM, Kim HS, Cashion A, Mysliwiec V, Diaz-Arrastia R, Gill J. Peripheral Total Tau in Military Personnel Who Sustain Traumatic Brain Injuries During Deployment. JAMA Neurol. 2015 Oct;72(10):110916. doi: 0.1001/jamaneurol.2015.1383;

Di Battista AP, Rhind SG, Richards D, Churchill N, Baker AJ, Hutchison MG Altered Blood Biomarker Profiles in Athletes with a History of Repetitive Head Impacts. PLoS One. 2016 Jul 26;11(7):e0159929. doi: 10.1371/journal.pone.0159929. Erratum in: PLoS One. 2016 Oct 11;11(10 ):e0164912;

Auvergne L, Bortsov AV, Ulirsch JC, Peak DA, Jones JS, Swor RA, Domeier RM, Lee DC, Rathlev NK, Hendry PL, McLean SA. Association of Epidemiologic Factors and Genetic Variants Influencing Hypothalamic-Pituitary-Adrenocortical Axis Function WithPostconcussive Symptoms After Minor Motor Vehicle Collision. Psychosom Med. 2016 Jan;78(1):68-78. doi: 10.1097/PSY.0000000000000253;

Graham MR, Pates J, Davies B, Cooper SM, Bhattacharya K, Evans PJ, Baker JS. Should an increase in cerebral neurochemicals following head kicks in full contact karate influence return to play? Int J ImmunopatholPharmacol. 2015 Dec;28(4):539-46. doi: 10.1177/0394632015577045;

Siman R, Shahim P, Tegner Y, Blennow K, Zetterberg H, Smith DH. J. Serum SNTF Increases in Concussed Professional Ice Hockey Players and Relates to the Severity of Postconcussion Symptoms. Neurotrauma. 2015 Sep 1;32(17):1294-300. doi: 10.1089/neu.2014.3698;

Schulte S, Podlog LW, Hamson-Utley JJ, StrathmannFG, Strüder HK. J Athl Train. A systematic review of the biomarker S100B: implications for sport-related concussion management. 2014 Nov-Dec;49(6):830-50. doi: 10.4085/1062-605049.3.33. Review;

Neselius S, Zetterberg H, Blennow K, Marcusson J, Brisby H. Increased CSF levels of phosphorylated neurofilament heavy protein following bout in amateur boxers. PLoS One. 2013 Nov 15;8(11):e81249. doi: 10.1371/journal.pone.0081249.

Seliyanina NV, Karakulova YV, Nenasheva OY. Klin Lab Diagn. The value of immune enzyme testing of qualitative content of blood serotonin and liquor, in differentiate diagnostic of craniocerebral injury]. May;(5):11-3. Russian. 2013; 
Tate CM, Wang KK, Eonta S, Zhang Y, Carr W, Tortella FC, Hayes RL, Kamimori GH. J Neurotrauma. Serum brain biomarker level, neurocognitive performance, and self-reported symptom changes in soldiers repeatedly exposed to low-level blast: a breacher pilot study. Oct 1;30(19):1620-30. doi: 10.1089/neu.2012.2683. 2013;

Kickboxing sport as a new cause of traumatic brain injury-mediated hypopituitarism.Tanriverdi F, Unluhizarci K, Coksevim B, Selcuklu A, Casanueva FF, Kelestimur F. Clin Endocrinol (Oxf). 2007 Mar;66(3):360-6;

Lu C-H, Allen K, Oei F, Leoni E, Kuhle J, Tree T, et al. Systemic inflammatory response and neuromuscular involvement in amyotrophic lateral sclerosis. NeurolNeuroimmunol neuroinflammation [Internet]. 2016;3(4):e244;

Bassini A, Cameron LC, Sportomics: building a new concept in metabolic studies and exercise science. BiochemBiophys Res Commun. 2014 Mar 21;445(4):708-16.;

Emery $\quad \mathrm{CA}^{1}$, Black $\quad \mathrm{AM}^{1}$, Kolstad $\quad \mathrm{A}^{1}$, Martinez $\quad \mathrm{G}^{1}$, Nettel-Aguirre $A^{1}$, Engebretsen $L^{2,3,4}$, Johnston $K^{5,6}$, Kissick $J^{7,8,9}$, Maddocks $D^{10}$, Tator $C^{11,12}$, Aubry $\mathrm{M}^{13,14,15}$, Dvořák $\mathrm{J}^{16,17}$, Nagahiro $\mathrm{S}^{18}$, Schneider $\mathrm{K}^{1}$. What strategies can be used to effectively reduce the risk of concussion in sport? A systematic review. $\mathrm{Br} \mathrm{J}$ Sports Med. 2017 Jun;51(12):978-984. doi: 10.1136/bjsports-2016-097452. Epub 2017 Mar;

Jin $X^{1}$, Feng $Z^{1}$, Mika $V^{1}$, Li $H^{1,2}$, Viano $\mathrm{DC}^{1,3}$, Yang $\mathrm{KH}^{1}$. The Role of Neck Muscle Activities on the Risk of Mild Traumatic Brain Injury in American Football. J Biomech Eng. 2017 Oct 1;139(10). doi: 10.1115/1.4037399;

Hrysomallis $\mathrm{C}^{1}$. Neck Muscular Strength, Training, Performance and Sport Injury Risk: A Review. Sports Med. 2016 Aug;46(8):1111-24. doi: 10.1007/s40279016-0490-4;

Theo Versteegh, Danielle Beaudet, Marla Greenbaum, Leah Hellyer, Amanda Tritton, Dave Walton. Evaluating the Reliability of a Novel NeckStrength Assessment Protocol for Healthy Adults Using Self-Generated Resistance with a Hand-Held Dynamometer. Physiother Can. 2015 Winter; 67(1): 5864. Published online 2015 Jan 27. doi: 10.3138/ptc.2013-66;

Falleti MG, Maruff P, Collie A, Darby DG. Practice effects associated with the repeated assessment of cognitive function using the CogState battery at 10-minute, one week and one month test-retest intervals. J ClinExpNeuropsychol2006;28:10951112;

Falleti MG, Maruff P, Collie A, Darby DG, McStephen M. Qualitative similarities in cognitive impairment associated with $24 \mathrm{~h}$ of sustained wakefulness and a blood alcohol concentration of 0.05\%. J Sleep Res 2003;12:265-274.

Cairney S, Clough AR, Maruff P, Collie A, Currie BJ, Currie J. Saccade and cognitive function in chronic kava users. Neuropsychopharmacology 2003;28:389396.

Brian H. Rowe, Leeor Eliyahu, Justin Lowes, Lindsay A. Gaudet, Jeremy Beach, Martin Mrazik, Garnet Cummings, Donald Voaklander, Concussion diagnoses among adults presenting to three Canadian emergency departments: Missed opportunities. Yajem (2017), doi:10.1016/j.ajem.2018.03.040

McCrory P, Meeuwisse W, Johnston K, et al. Consensus statement on concussion in sport: the 3rd International Conference on Concussion in Sport held in Zurich, November 2008. J Athl Train. 2009;44(4):434-448. doi:10.4085/1062-605044.4.434 
Neto, MS; Lobo, PL; Oliveira, M; Albuquerque, ACS; Crema, PH. FIFA Medical Centre of Excellence Meeting, London, 28 April. 2019. 
ANEXO 1

N. of concussions per athlete

1

70

2

20

3

5

4

2.5

5

2.5 
ANEXO 2 - Incidência de Lesões

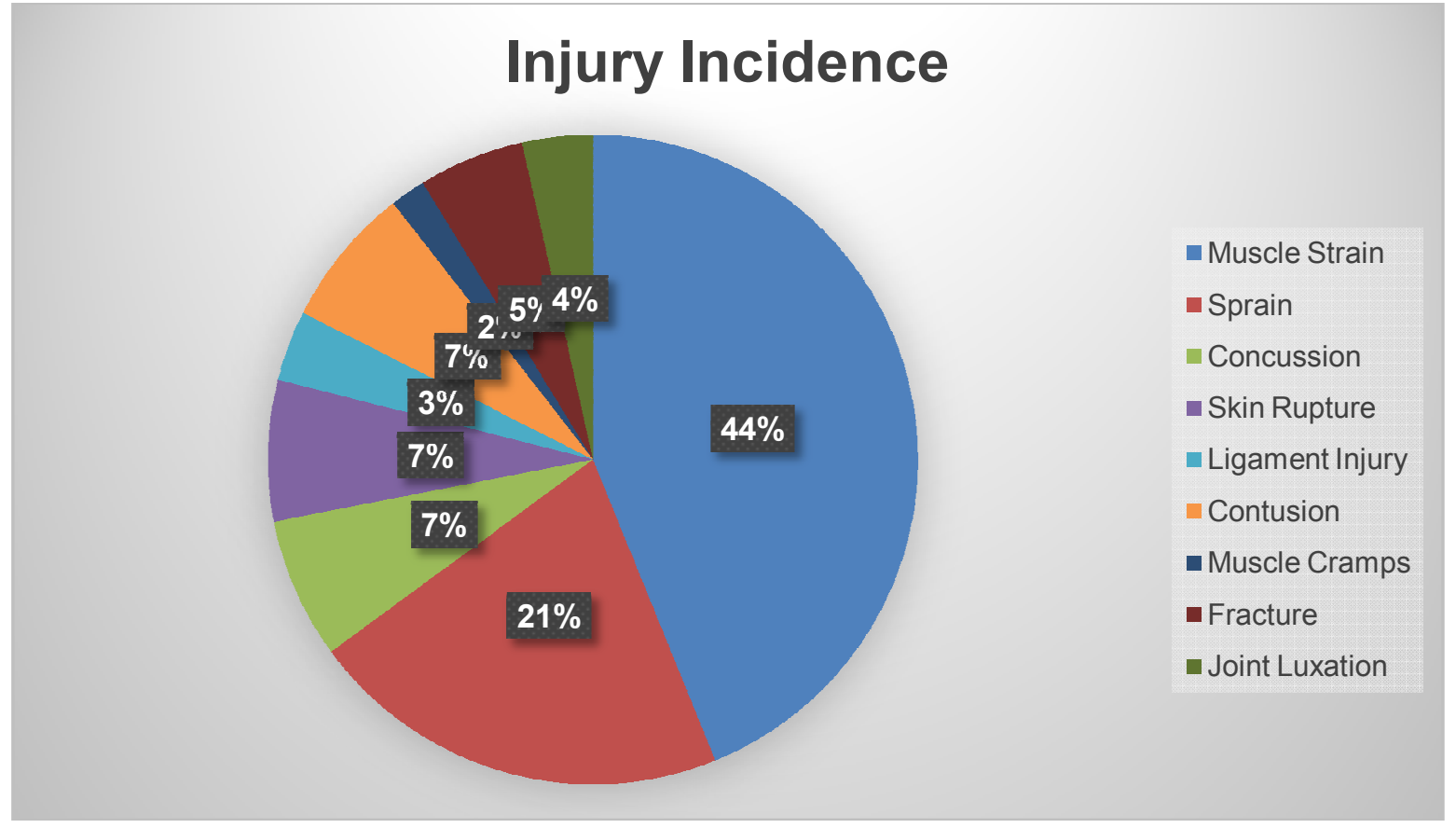

